\title{
Tuberculosis ganglionar cervical. ¿Pensamos en ella, o nos sorprende?
}

\section{Neck node tuberculosis. Do we consider it... or does it pop up?}

\author{
Miguel Alberto Rodríguez-Pérez', Fernando Aguirre-García².
}

\section{RESUMEN}

Revisamos las características clínicas, diagnóstico y manejo de la tuberculosis (TB) cervical, así como resaltamos su importancia por su carácter epidémico.

Presentamos dos pacientes afectados por tumoraciones laterocervicales subagudas, escasa sintomatología y excelente evolución tras su diagnóstico de TB ganglionar cervical y tratamiento antibiótico.

$L a T B$ es una enfermedad que en la actual sociedad globalizada, puede encontrarse prácticamente cualquier especialista, por lo que debemos mantener un alto nivel de alerta y conocerla con detalle, para poder orientar su diagnóstico y facilitar su tratamiento precoz.

Palabras clave: Tuberculosis, adenopatías, cuello, micobacteria, linfadenitis.

\begin{abstract}
We review tuberculosis clinical features, diagnosis and management as well as remark the importance of its epidemic nature.

Study based on 2 patients suffering from eye-catching subacute neck lumps, sparsely symptomatic and excellent evolution after neck node TB diagnosis and antibiotic therapy.

$T B$ represents a disease that, given our present time globalization, may be faced by many different specialists. That is why we must be on alert and be aware of its profile, in order to guess the right diagnosis and offer therapy.
\end{abstract}

Key words: Tuberculosis, nodes, neck, mycobateria, lymphadenitis.

1 Unidad de Gestión Clínica de Otorrinolaringología, Hospital Universitario de Puerto Real (Cádiz, España).

2 Servicio de Otorrinolaringología, Hospital “Virgen del Puerto”, Plasencia, Cáceres, España. 


\section{INTRODUCCIÓN}

La tuberculosis (TB) es una enfermedad granulomatosa crónica provocada por Mycobacterium tuberculosis y que puede afectar a cualquier órgano, transmitida fundamentalmente a través del tracto respiratorio.

Ya se conocen descripciones en la Grecia clásica, y fue nombrada con "el mal de los reyes" en la Edad Media, o "escrófula" cuando Koch descubre y presenta su etiología micobacteriana en su forma ganglionar cervical en el siglo XIX.

En las últimas décadas se había producido un descenso en su incidencia en los países occidentales, interrumpido por la expansión del Síndrome de Inmunodeficiencia Adquirida (SIDA) en los años ' 90 , y más recientemente por los casos importados por inmigrantes ${ }^{1}$.

Se trata de un problema de salud global, con casi 8 millones de personas afectadas/año (India aporta casi un tercio de esta cantidad, con una prevalencia de 4,4 casos/ 1.000 habitantes menores de 14 años, e incidencia de 168/100.000 personas/año, 42 veces mayor que la de EE.UU. $)^{2,3}$ y 3 millones de personas/año muriendo por sus complicaciones ${ }^{4}$. Es la mayor causa de muerte por un único agente infeccioso, y su creciente incidencia, especialmente en países subdesarrollados, ha hecho que la Organización Mundial de la Salud (OMS) la haya declarado una emergencia global ${ }^{5}$.
Actualmente la TB extrapulmonar constituye el $25 \%$ del total de la patología TB, del cual la afectación ganglionar cervical constituye el 35\%-50\%, lo cual la convierte en una entidad que debe ser conocida y sospechada, a pesar de caracterizarse por ser algo imitadora, y poder coexistir o enmascararse con otras patologías cérvico-faciales de carácter benigno o maligno, pues obteniendo un mayor índice de sospecha podrían orientarse los diagnósticos de forma más precoz y permitir tratamientos más acer$\operatorname{tados}^{1,3,6}$.

El objetivo perseguido en este estudio es revisar las características clínicas y el manejo de la TB cervical, e identificar aquellos rasgos que puedan orientar su diagnóstico, así como concederle la importancia que merece por su implicación como epidemia.

Presentamos 2 pacientes estudiados y tratados de nuestra patología objeto de presentación por el Servicio ORL del Hospital "Virgen del Puerto" de Plasencia (Cáceres, España), entre los años 2006 y 2010.

\section{CASOS CLÍNICOS}

Paciente 1. Mujer de 84 años, sin antecedentes de interés, estudiada en consultas de ORL por una tumoración de región parotídea izquierda, dolorosa y surgida de forma subaguda en 1 mes de evolución, sin más clínica. Figura 1.

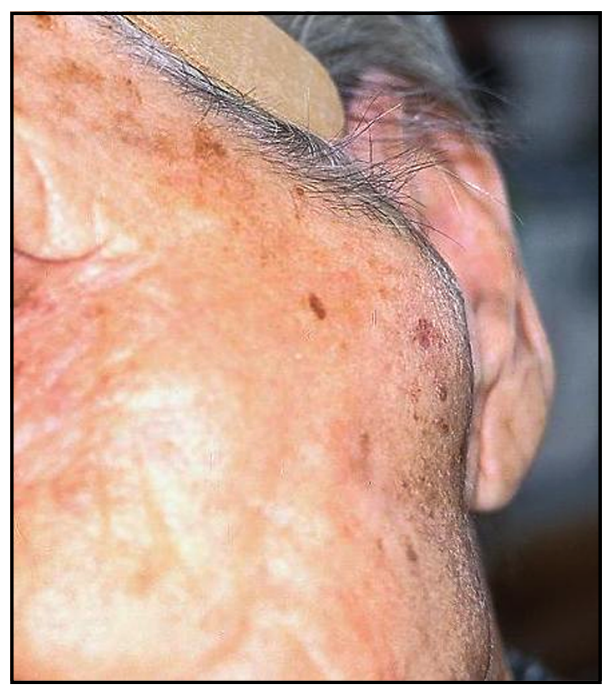

Figura 1. Tumoración laterocervical. 
A la inspección podía apreciarse una lesión redondeada de $4 \times 3 \mathrm{~cm}$, con una palpación fluctuante, elástica, dolorosa, no signos inflamatorios, protruyendo sobre CAE izquierdo. Se realizó punción/drenaje de la misma obteniendo material caseoso espeso verde-amarillento. El resto de la exploración ORL fue normal.

PAAF: "no concluyente".

Anatomía patológica: "Proceso inflamatorio crónico granulomatoso necrotizante. Tinción de Ziehl para bacilo ácido-alcohol resistente positiva".

Microbiología: "Auramina positiva".

Radiografía ántero-posterior y lateral de tórax: "Sin hallazgos destacables".

TC cervical: "Masa bien delimitada ligeramente hipodensa de $5 \times 3,5 \mathrm{~cm}$ situada en espacio masticador izquierdo. Afecta lateralmente al músculo masetero y medialmente al pterigoideo lateral, produciendo efecto masa sobre el espacio parafaríngeo. Fosa infratemporal izquierda ocupada, sin invasión de fosa pterigopalatina. Posteriormente llega a pared anterior de CAE invade la articulación temporomandibular con osteolisis del cóndilo mandibular. No se aprecian adenopatías".

Una vez obtenido el diagnóstico AP, se inició tratamiento antituberculoso con isoniazida, rifampicina y pirazinamida durante 2 meses, manteniendo otros 4 meses más los 2 primeros. La evolución fue excelente, aunque durante 3-4 meses pre- cisó varias evacuaciones de contenido purulento local.

Paciente 2. Mujer de 85 años sin ningún antecedente destacable, seguida desde urgencias por una tumoración en región laterocervical izquierda posterior, de 2 meses de evolución, de crecimiento progresivo y dolorosa, acompañada de fiebre ocasional. Sin otra sintomatología.

La exploración ORL muestra tumoraciones laterocervicales múltiples, la mayor de unos $3 \times 3 \mathrm{~cm}$ de diámetro, profundas, dolorosas, fluctuantes y fijas. Figura 2.

ECO cervical: "Múltiples adenopatías laterocervicales izquierdas de ecoestructura mixta, probablemente masa adenopática en fase flegmonosa".

PAAF: "No concluyente".

TC cervical: "Masa hipodensa de bordes lobulados y captación periférica látero-cervical superior, submandibular posterior-izquierda y supraclavicular izquierda, compatibles con masas de conglomerados adenopáticos con necrosis central, de probable origen linfoproliferativo, sin poder descartar proceso infeccioso: TB":

Tinción de Ziehl-Neelsen (BAAR): Positiva para TB.

Se decide realizar cervicotomía para obtener material para estudio anatomopatológico y microbiológico, con el resultado de positividad para infección ganglionar por Mycobacterium tuberculosis.

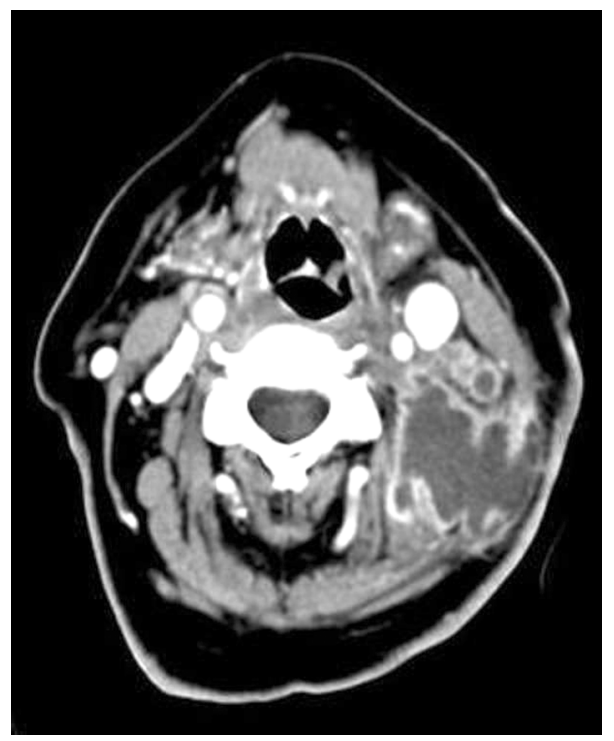

Figura 2. TC cervical. 
El tratamiento seguido fue el mismo que en el caso de la paciente 1 .

\section{DISCUSIÓN}

Clásicamente la linfadenitis cervical tuberculosa (la región de cabeza y cuello afectada más frecuentemente por la TB extrapulmonar) ha sido una patología más común en niños y adultos jóvenes (1,3 millones de casos y 450.000 muertes/año suceden en niños) ${ }^{7}$ pero el pico de edad de mayor incidencia se ha desplazado a los 20-40 años, y con una frecuencia del $70 \%$ en sexo femenino ${ }^{5}$.

En países desarrollados la mayoría de casos ocurren en población adulta inmigrante de regiones endémicas ( $70 \%$ en Francia y Alemania) $)^{5}$.

La afectación ganglionar cervical tuberculosa supone el $5 \%$ de los procesos linfadenopáticos de dicha zona, siendo el triángulo posterior, el supraclavicular y la cadena de la vena yugular interna los afectados más comúnmente ${ }^{3,6}$.

La TB es 500 veces más frecuente en pacientes con HIV que en la población sana, con hasta $80 \%$ de la población afectada desarrollando patología extrapulmonar².

En cualquier caso, también debemos contar como de alto riesgo a la población envejecida e inmunodeprimidos no infectados por HIV como trasplantados, tratados con inmunosupresores, cirróticos o nefrópatas, y saber que el hacinamiento y los focos de pobreza crecientes en las grandes ciudades también son predisponentes y facilitan la difusión.

Tras la afectación ganglionar ( $95 \%$ de casos) ${ }^{1}$, encontramos la laríngea en forma de úlceras de cuerdas vocales o nódulos/pólipos epiglóticos (es destacable recordar que hasta el s. XX fue la enfermedad laríngea más frecuente: hasta el $25 \%$ de la población general); y con menor frecuencia en área nasal/paranasal, oral, glándulas salivares, vértebras cervicales y pabellones auditivos/mastoides ${ }^{3}$.

Es necesario destacar que no es frecuente la afectación pulmonar simultánea en estos pacientes, sólo en 10\%-20\% de ellos, pero sí existe un alto nivel de coexistencia con infección por $\mathrm{VIH}^{3}$.

La vía de infección más probable es vía aérea por gotas de Pflüger que provoca el contacto con el anillo de Waldeyer, y desde éste, diseminación y afec- tación secundaria local. Se piensa que podría ser consecuencia de una reactivación de una infección previa en el tiempo, quizás años atrás.

La inoculación directa desde un foco pulmonar a laringe, faringe y cavidad oral es posible mediante expectoración de esputo infeccioso, así como la llegada del bacilo vía hematógena o linfática a los ganglios.

La presentación clínica más frecuente se acoge a la descrita con nuestras pacientes: masa cervical multilobulada unilateral $(66 \%-80 \%)^{1,6}$, consistente en adenopatías múltiples aglomeradas y abscedadas, fiebre nocturna, anorexia, astenia, pérdida de peso $y$ dolor aparecen en el $50 \%$ de los casos, y que se mantienen durante semanas o meses antes de llegar al diagnóstico. Así, el diagnóstico diferencial frente a linfoma o metástasis puede ser complicado únicamente con la exploración clínica.

El proceso diagnóstico podemos iniciarlo con la PAAF, pero el definitivo debe ser una combinación de estudio microbiológico y anatomopatológico, teniendo en cuenta que muchas veces se debe plantear una intervención quirúrgica para obtención de material más satisfactorio.

La PAAF es simple, segura, con pocas complicaciones y coste-efectiva, presentando una especificidad del $93 \%$ y una sensibilidad del $77 \%{ }^{3}$.

Es precisa para el diagnóstico definitivo la verificación mediante cultivo microbiológico en medios específicos, así como para conocer posibles resistencias a antibióticos. Sin embargo, un cultivo negativo no excluye la enfermedad porque los ganglios podrían no contener bacilos vivos, por lo que tiene un rendimiento limitado, y además sus resultados pueden tardar semanas en llegar, y el paciente probablemente ya esté en tratamiento. Es por ello que quizás sea buena opción la PAAF inicial como método más rápido.

La radiografía de tórax debe ser realizada de forma invariable para descartar la afectación pulmonar.

La imagen que nos ofrece la ecografía son nódulos hipoecoicos con un patrón heterogéneo y necrosis central, junto a edema del tejido blando perilesional.

Para conocer la disposición y grado de afectación tisular y anatómica de la región contamos con la tomografía computarizada, que nos permite conocer la morfología de las lesiones de forma deta- 
llada, con vistas a una posible cirugía. Lo característico es la formación de conglomerados, hipodensidad central del nódulo, con realce periférico e inflamación del tejido blando circundante.

La Resonancia Magnética Nuclear también puede sernos de ayuda en la evaluación de la afectación locorregional.

En la anatomía patológica veremos granulomas con células gigantes multinucleadas e infiltración aguda neutrofílica, con 0 sin necrosis.

Métodos de cultivo rápido han posibilitado la identificación positiva e inicio del tratamiento precoz conociendo la sensibilidad al antimicrobiano. Por otro lado, la detección rápida de micobacterias por amplificación del ADN (reacción en cadena de la polimerasa) son de ayuda en casos de dificultad diagnóstica. Se calcula una sensibilidad de muy variable, $41 \%-75 \%$, y especificidad de casi $100 \%$, con gran variabilidad según el laboratorio¹.

Los diagnósticos diferenciales frente a los cuales tenemos que plantearla incluirían el linfoma, el carcinoma epidermoide de cabeza y cuello de forma primaria o metastásica, carcinoma papilar tiroideo, procesos inflamatorios y granulomatosos como la enfermedad de Wegener, lupus eritematoso sistémico, sarcoidosis, artritis reumatoide, amiloidosis, policondritis, enfermedad de Castleman, sífilis, enfermedad por arañazo de gato, toxoplasmosis, actinomicosis, tularemia, infecciones víricas sistémicas (mononucleosis).

Por supuesto debemos conocer si el paciente reside o procede de áreas endémicas, 0 si ha estado en contacto 0 expuesto a la enfermedad en el pasado cercano o lejano.

Actualmente el tratamiento antibiótico es la opción indicada, con $80 \%-90 \%$ de éxito ${ }^{6,9}$, con la pauta ya indicada de isoniazida, rifampicina y pirazinamida durante 2 meses, añadiendo otros 4 meses con sólo los 2 primeros; mientras que la cirugía, opción controvertida, se reserva a aquellos casos de grandes conglomerados adenopáticos 0 abscesos fríos, o bien cuando es precisa una biopsia (siempre excisional y nunca realizando linfadenectomías amplias), porque el diagnóstico definitivo no se ha podido obtener de otro modo (habitualmente por citologías no determinantes).

Podemos encontrar tasas de recidiva de 3,5\%, 0 de persistencia tras la terapia de $7 \%-11 \%{ }^{1}$.
Debemos controlar la hepatotoxicidad e interacciones con otros fármacos, así como ser conscientes de la posibilidad de una reacción paradójica (20\% de casos, pero mayor en grupo de $\mathrm{VIH+}$, que provoca crecimiento ganglionar, fluctuación, dolor e incluso fístula al inicio del tratamiento. Puede requerir drenajes repetidos y tratamiento corticoideo.

Con la cirugía podríamos provocar la diseminación locorregional de la enfermedad, así como la aparición de complicaciones del tipo fístula crónica, retrasos de la cicatrización, cicatrices deformantes, síndrome de Horner, lesiones de VIIpc, XIpc.

En el siglo XXI debemos entender que vamos a conocer a una TB diferente, con afectaciones orgánicas únicas, problemas asociados a su coexistencia con el HIV y un aumento progresivo de las multirresistencias a los antimicrobianos.

De esta forma, inicialmente su diagnóstico se convierte en un reto para el médico, que debe mantenerse actualizado y alerta ante la mínima sospecha, para posteriormente enfrentarse al desafío del tratamiento que debe cumplir los objetivos de la curación y de frenar la transmisión.

Sería ideal plantearse el diagnóstico antes de enfrentarnos a una patología orientada hacia otra patología y que no responde al tratamiento convencional antibiótico 0 antiinflamatorio, o bien en lesiones con apariencia inusual o que no manifiestan malignidad. Es necesario un alto índice de sospecha en pacientes inmunocomprometidos 0 inmersos en un entorno socioeconómico desfavorecido.

Además, son múltiples las especialidades médico-quirúrgicas que pueden enfrentarse a este tipo de pacientes, y hasta el más experimentado podría verse sorprendido, de forma que debe ser una entidad a tener en cuenta permanentemente, más aún cuando la globalización que nos afecta en todos los ámbitos nos convierte en dianas potenciales a cada uno de nosotros, permite que las migraciones, el turismo, la alimentación y el contacto cultural e interpersonal se conviertan en perfectos "vectores" de la TB.

Un tratamiento precoz previene complicaciones y la morbilidad de la enfermedad diseminada. 


\section{BIBLIOGRAFÍA}

1. Peralta Fernández, G. Tuberculosis de cabeza y cuello. Acta Otorrinolaringol Esp 2009; 60(1): 59-66.

2. Prasad KC, Sreedharan S, Chakravarthy Y. Tuberculosis in the head and neck: experience in India. The Journal of Laryngology \& Otology 2007; 121: 979-85.

3. Vaid S; Lee YyP; Rawat S; Luthra A. Tuberculosis in the head and neck-a forgotten differential diagnosis. Clinical Radiology 2010; 65: 73-81.

4. Wang WC; Chen JY; Chen YK. Tuberculosis of the head and neck: a review of 20 cases. Oral Surg Oral Med Oral Pathol Oral Radiol Endod 2009; 107: 381-6.

5. Choudhury N; Bruch G; Kothari P; Rao, G. 4 years' experience of head and neck tuberculosis in a south London hospital. J R Soc Med 2005; 98: 267-9.

6. Ammari FF; Bani Hani AH; Ghariebeh KI. Tuberculosis of the lymph glands of the neck: a limited role for surgery. Otolaryngol Head Neck Surg 2003; 128: 576-80.

7. Surer I; Ozturk H; Cetinkursun S. Unusual presentation of tuberculosis reactivation in childhood: an anterior neck mass. J Pediatr Surg 2000; 35: 1263-5.

8. Singh KK; Muralihdar M; Kumar A. Comparison of in house polymerase chain reaction with conventional techniques for the detection of mycobacterium tuberculosis DNA in granulomatous lymphadenopathy. J Clin Pathol 2000; 53: 355.

9. Benhammou A; El-Ayoubi A; Benbouzid MA. Tuberculosis primaire de la glande parotide. Archives de Pédiatrie 2007; 14: 1206-9. 